\title{
Using Least Squares to Improve Blind Signal Copy Performance
}

\author{
A. Swindlehurst, Member, IEEE, and J. Yang
}

\begin{abstract}
Conventional methods for signal copy require one to estimate the directions of arrival (DOA's) of the signals prior to computing the weight vectors. Blind copy algorithms alleviate the need for DOA estimation (and hence the need for array calibration data) by exploiting the temporal rather than spatial structure of the signals, but they converge slowly in some cases. In this letter, we present a simple technique that uses both spatial and temporal information to improve signal copy performance. Specifically, the algorithm uses an initial blind estimate of the signals to compute a least-squares estimate of the array response, which in turn is used to update the signal estimates.
\end{abstract}

\section{INTRODUCTION}

C ONVENTIONAL techniques for signal copy such as least-squares (LS) that exploit the spatial coherence of the incoming signals cannot be applied without accurate knowledge of the directions of arrival (DOA's) of the signals and the array geometry or response. In addition, such techniques make no assumption about the signals themselves and consequently cannot exploit any such available information. In response to these drawbacks, so-called blind copy algorithms such as SCORE [1], [2], the constant modulus approach (CMA) [3], [4], and various property restoral algorithms [5] have been proposed. These methods exploit known properties about the signals (cyclostationarity, constant modulus, etc.) to alleviate the need for precise array calibration data. On the other hand, these algorithms may converge slowly in certain situations (e.g., if it is feasible to exploit only baud rate and not carrier frequency features), and they have difficulty with certain types of signal scenarios (e.g., correlated signals).

Thus, conventional techniques based on direction finding (DF) do not employ any knowledge about the signal structure, and blind copy algorithms do not use any knowledge of the array response. In many situations, information about both the array calibration and the signal structure is available (or can be estimated from the data), and it is obviously suboptimal to ignore either. Although a criterion function could be developed and jointly minimized with respect to both the signal waveforms and DOA's (with the available prior information acting as a constraint on the minimization), it is likely that such an approach would be prohibitively complex. As an alternative, we present a simple suboptimal

Manuscript received April 4, 1994; approved April 7, 1994. This work was supported by a contract from E-Systems, Inc., Greenville Division (Dr. W. A. Gardner, Principal Investigator), and by the National Science Foundation under grant MIP-9110112. The associate editor coordinating the review of this letter and approving it for publication was Prof. J. Moura.

The authors are with the Department of Electrical and Computer Engineering, Brigham Young University, Provo, UT 84602 USA

IEEE Log Number 9402222. algorithm that attempts to improve signal copy performance by exploiting both the array and signal structure in an alternating fashion.

Similar "alternating projection" techniques have been proposed elsewhere. In [1], an adaptive implementation of SCORE was presented where one alternates between estimating the signal copy weights and the Cross-SCORE control vector. The LS-CMA approach described in [5] and [6] alternates between estimating the signals and using a modulusrestored version of the signals to generate new signal copy weights and typically converges much more quickly than conventional CMA. The algorithm we propose is different in that it attempts to incorporate spatial information from the array by estimating its response from the initial signal estimates. In particular, our method assumes an arbitrary blind copy algorithm has been applied to obtain an initial estimate of the signal waveforms and then uses LS methods to estimate the steering matrix and revise the signal estimates.

\section{DATA MODEL}

Consider an array of $m$ sensors having arbitrary positions and characteristics that receives the waveforms of $d$ narrowband (co-channel) signals. The vector of complex sensor outputs is denoted $\mathbf{x}(t)$ and is modeled by the following familiar equation:

$$
\begin{aligned}
\mathbf{x}(t) & =\left[\mathbf{a}\left(\theta_{1}\right)|\cdots| \mathbf{a}\left(\theta_{d}\right)\right]\left[\begin{array}{c}
s_{1}(t) \\
\vdots \\
s_{d}(t)
\end{array}\right]+\mathbf{n}(t) \\
& =\mathbf{A}(\theta) \mathbf{s}(t)+\mathbf{n}(t) .
\end{aligned}
$$

The columns of the $m \times d$ matrix $\mathbf{A}$ are the so-called steering or propagation vectors of the array and are denoted as $\mathbf{a}\left(\theta_{i}\right), i=1, \ldots, d$. These vectors are functions of the signal parameters and describe the array response to a unit waveform with parameter(s) $\theta_{i}$. Although not necessary, we will assume that $\theta_{i}$ is a real-valued scalar referred to as the $i$ th DOA (in general, the response for a given signal could depend on more than one parameter). The $d$ vector $\mathbf{s}(t)$ is composed of the complex waveforms (in-phase and quadrature components) of the signals received at time $t$, and the $m$ vector $\mathbf{n}(t)$ accounts for additive measurement noise. The noise term is assumed to be spatially white and uncorrelated with the signals.

The general problem addressed in this paper is the estimation of the signal waveforms at $N$ distinct sample points $\mathbf{S}=[\mathbf{s}(1), \cdots, \mathbf{s}(N)]$ using the received data $\mathbf{X}$ :

$$
\mathbf{X}=\mathbf{A}(\theta) \mathbf{S}+\mathbf{N}
$$


where $\mathbf{X}$ and $\mathbf{N}$ are defined similarly to $\mathbf{S}$. This is typically done by forming a linear combination of the array outputs as in

$$
\hat{\mathbf{S}}=\mathbf{W}^{*} \mathbf{X} \text {. }
$$

The $i$ th column of the matrix $\mathbf{W}$ is referred to as the signal copy weight vector (or sometimes beamformer weights) for the $i$ th signal. There are a number of methods available for choosing the weight matrix $\mathbf{W}$, each with a different optimality criterion and a different set of assumptions about what a priori information is available. In the LS approach, one finds the signal estimate that in the LS sense, best matches the received data given an estimate of the steering matrix $\hat{\mathbf{A}}$ :

$$
\begin{aligned}
\hat{\mathbf{S}}_{\mathrm{LS}} & =\arg \min _{S}\|\mathbf{X}-\hat{\mathbf{A}} \mathbf{S}\|_{F}^{2} \\
& =\hat{\mathbf{A}}^{\dagger} \mathbf{X},
\end{aligned}
$$

where in this case $\mathbf{W}_{\mathrm{LS}}^{*}=\hat{\mathbf{A}}^{\dagger}=\left(\hat{\mathbf{A}}^{*} \hat{\mathbf{A}}\right)^{-1} \hat{\mathbf{A}}^{*}$. Although $\hat{\mathbf{A}}=\mathbf{A}(\hat{\theta})$ is typically obtained by first estimating the DOA's, this is not necessary since there is no explicit dependence on the DOA's in (5). In particular, if $\mathbf{S}$ were known (or an estimate $\hat{\mathbf{S}}$ were available), one could minimize (4) with respect to $\hat{\mathbf{A}}$ to obtain

$$
\begin{aligned}
& \hat{\mathbf{A}}=\mathbf{X S}^{*}\left(\mathbf{S S}^{*}\right)^{-1}=\mathbf{X} \mathbf{S}^{\dagger} . \\
& \text { III. A BLINDUS ALGORITHM }
\end{aligned}
$$

\section{A BLINDUS ALGORITHM}

An outline of the algorithm we propose is given below:

1. Use a blind copy algorithm to find a set of signal copy weights $\mathbf{W}$, and obtain an initial signal estimate

$$
\hat{\mathbf{S}}=\mathbf{W}^{*} \mathbf{X} \text {. }
$$

2. Treating $\hat{\mathbf{S}}$ as a constant, minimize the $\mathbf{L S}$ cost function $\|\mathbf{X}-\mathbf{A} \hat{\mathbf{S}}\|_{F}^{2}$ with respect to $\mathbf{A}$ to obtain the following estimate of the steering matrix:

$$
\hat{\mathbf{A}}=\mathbf{X} \hat{\mathbf{S}}^{\dagger} \text {. }
$$

3. Treating $\hat{\mathbf{A}}$ as a constant, obtain a refined estimate of the signals using LS again:

$$
\hat{\hat{\mathbf{S}}}=\hat{\mathbf{A}}^{\dagger} \mathbf{X}
$$

If we assume that $\tilde{\mathbf{S}}$ represents the error associated with the initial blind signal estimate $(\hat{\mathbf{S}}=\mathbf{S}+\tilde{\mathbf{S}})$, then the resulting error in the estimate of $\mathbf{A}$, which is denoted $\Delta \mathbf{A}$, can be approximated as

$$
\begin{aligned}
\hat{\mathbf{A}}=\mathbf{A}+\Delta \mathbf{A} & =(\mathbf{A S}+\mathbf{N}) \hat{\mathbf{S}^{*}}\left(\hat{\mathbf{S}} \hat{\mathbf{S}}^{*}\right)^{-1} \\
& \simeq \mathbf{A}-\mathbf{A} \tilde{\mathbf{S}} \mathbf{S}^{\dagger}+\mathbf{N} \tilde{\mathbf{S}}^{*}\left(\mathbf{S S}^{*}\right)^{-1}
\end{aligned}
$$

to first order in $\tilde{\mathbf{S}}$. Note that we have also ignored terms involving the product $\mathrm{NS}^{*}$ since the signals and noise are assumed to be uncorrelated. Using similar first-order arguments, the refined signal copy estimate may be approximately expressed as

$$
\begin{aligned}
\hat{\hat{\mathbf{S}}}= & {\left[(\mathbf{A}+\mathbf{\Delta} \mathbf{A})^{*}(\mathbf{A}+\boldsymbol{\Delta} \mathbf{A})\right]^{-1}(\mathbf{A}+\boldsymbol{\Delta} \mathbf{A})^{*}(\mathbf{A} \mathbf{S}+\mathbf{N}) } \\
\simeq & \mathbf{S}+\mathbf{A}^{\dagger} \mathbf{N}+\left(\mathbf{A}^{*} \mathbf{A}\right)^{-1} \mathbf{\Delta} \mathbf{A}^{*} \mathbf{N}-\mathbf{A}^{\dagger} \mathbf{\Delta} \mathbf{A S} \\
& -\left(\mathbf{A}^{*} \mathbf{A}\right)^{-1}\left(\boldsymbol{\Delta} \mathbf{A}^{*} \mathbf{A}+\mathbf{A}^{*} \mathbf{\Delta} \mathbf{A}\right) \mathbf{A}^{\dagger} \mathbf{N}
\end{aligned}
$$

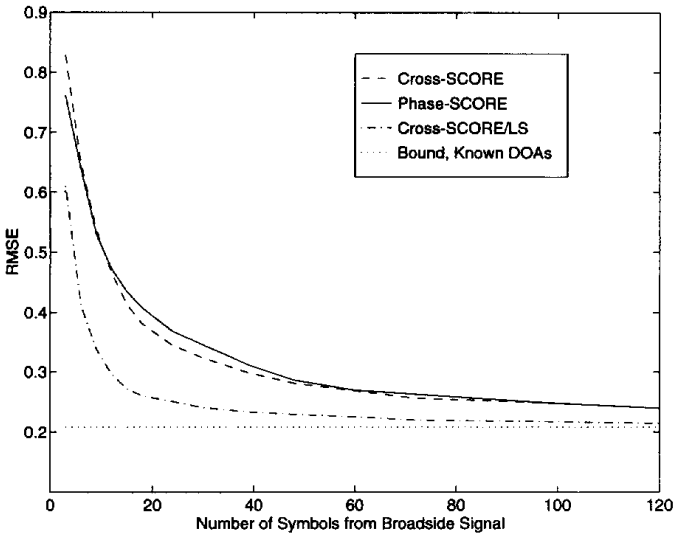

Fig. 1. Performance of the blind/LS algorithm versus Cross-SCORE.

Therefore, the final error in the signal copy is

$\tilde{\tilde{\mathbf{S}}} \simeq \mathbf{A}^{\dagger} \mathbf{N}+\left(\mathbf{A}^{*} \mathbf{A}\right)^{-1} \boldsymbol{\Delta} \mathbf{A}^{*} \mathbf{P}_{\boldsymbol{A}}^{\perp} \mathbf{N}-\mathbf{A}^{\dagger} \boldsymbol{\Delta} \mathbf{A} \mathbf{S}-\mathbf{A}^{\dagger} \boldsymbol{\Delta} \mathbf{A} \mathbf{A}^{\dagger} \mathbf{N}$

where $\mathbf{P}_{A}^{\perp}=\mathbf{I}-\mathbf{A}\left(\mathbf{A}^{*} \mathbf{A}\right)^{-1} \mathbf{A}^{*}$.

The final signal copy error thus depends on the initial error in arather complicated way. If we take the special case where the SNR is relatively high and the terms involving $\mathbf{N}$ above may be ignored, substituting (8) into (9) leads to

$$
\tilde{\tilde{\mathbf{S}}} \simeq-\mathbf{A}^{\dagger} \Delta \mathbf{A S} \simeq \tilde{\mathbf{S}} \mathbf{P}_{S^{*}}
$$

where $\mathbf{P}_{S^{*}}$ is the projection onto the row space of $\mathbf{S}$. This implies that $\|\tilde{\tilde{\mathbf{S}}}\| \leq\|\tilde{\mathbf{S}}\|$ and, hence, that the LS iteration in steps 2 and 3 will improve the MSE of the signal estimate. Consequently, in high SNR environments, repeating steps 2 and 3 will not lead to further improvement since to first order

$$
\tilde{\tilde{\mathbf{S}}} \simeq \tilde{\tilde{\mathbf{S}}} \mathbf{P}_{S^{*}} \simeq \tilde{\mathbf{S}} \mathbf{P}_{S^{*}} \mathbf{P}_{S^{*}}=\tilde{\mathbf{S}} \mathbf{P}_{S^{*}} \simeq \tilde{\tilde{\mathbf{S}}}
$$

\section{A Simulation EXAMPLE}

In this example, a six-sensor $\lambda / 2$ uniform linear array (ULA) with two 10-dB SNR signals incident from $\left[0^{\circ}, 12^{\circ}\right]$ was simulated. Both signals were BPSK with full raised-cosine pulse shapes and baud rates of four and six samples per symbol, respectively, and hence, both signals were uncorrelated. An initial blind estimate of the signals was generated using Cross-SCORE and the baud rate features of the signals. The root-MSE of Cross-SCORE based on 100 independent trials is plotted in Fig. 1 (dashed line) as a function of the amount of data used to calculate the weight vectors. The results for the Phase-SCORE algorithm (solid line) [1] are also plotted for reference. ${ }^{1}$ The dash-dot curve shows the root-MSE that results from refining the initial Cross-SCORE estimate using one iteration of the LS steps described by (6) and (7). The dotted line shows the theoretical root-MSE performance of the LS algorithm assuming that the array manifold and the

${ }^{1}$ The SCORE delay parameter $\tau$ was chosen to be zero for both Phase and Cross-SCORE since this yielded the best performance. 
DOA's of the signals are perfectly known (e.g., see [7]). Note that we are able to achieve performance comparable to the ideal known-array-response case even though this information was not used by the algorithm. As expected, the performance gain between Cross-SCORE alone and the LS refinements is greatest for small data sets, and the improvement decreases as Cross-SCORE is able to converge towards its limiting solution.

\section{REFERENCES}

[1] B. Agee, S. Schell, and W. Gardner, "Spectral self-coherence restoral: A new approach to blind adaptive signal extraction using antenna arrays," Proc. IEEE, vol. 78, no. 4, pp. 753-767, Apr. 1990.
[2] S. Schell, "An overview of sensor array processing for cyclostationary signals," in Cyclostationarity in Communications and Signal Processing. New York: IEEE, 1993.

[3] J. Treichler and B. Agee (W. A. Gardner, Ed.), "A new approach to multipath correction of constant modulus signals," IEEE Trans. Acoust. Speech Signal Processing, vol. ASSP-31, no. 2, pp. 459-472, Apr. 1983.

[4] R. Gooch and J. Lundel, "The CM array: An adaptive beamformer for constant modulus signals," in Proc. IEEE ICASSP, 1986, pp. 2523-2526.

[5] B. Agee, "Maximum likelihood approaches to blind adaptive signal extraction using narrowband arrays," in Proc. 25th Asilomar Conf. Signals Syst. Comput. (Asilomar, CA), Nov. 1991, pp. 716-720.

[6] _ "The least-squares CMA: A new technique for rapid correction of constant modulus signals," in Proc. IEEE ICASSP (Tokyo, Japan), 1986, pp. 953-956.

[7] J. Yang and A. Swindlehurst, "Signal copy with array calibration errors," in Proc. 27th Asilomar Conf. Signals Syst. Comput. (Asilomar, CA), Nov. 1993, pp. 1533-1537. 